\title{
Surgical Complications in Early Period after Renal Transplantation
}

\author{
Janis Jushinskis, Vadims Suhorukovs, Sergejs Trushkovs, Janis Bicans, Victors Shevelevs, Rafails Rozentals \\ Riga Stradins University, Transplant Laboratory \\ Pauls Stradins Clinical University Hospital, Latvian Transplantation Center
}

\begin{abstract}
Summary.
Introduction. Growing number of elderly transplant recipients and expansion of the criteria for organ donation may increase the risk of post-transplant complications and impact outcomes.

Aim of the Study. The aim of this study was to define the rate of surgical complications needing re-operations and their impact on posttransplant outcomes.

Materials and methods. Study includes 202 consecutive deceased donor renal transplantations performed from 01.01 .2004 till 31.12.2006. with further follow-up for 3 years. We analyzed the rate of re-operations, associated donor, recipient and transplantation factors and impact on post-transplant outcomes.

Results. Reasons for re-operations were bleeding and hematoma formation ( $n=27)$, urological complications ( $n=18)$ and lymphocele $(n=26)$. Hematomas were associated increased donor body mass index (BMI, $p=0,067)$, presence of glomerular sclerosis at zerotime biopsy $(p=0,034)$ and with development of urological complications $(p=0,004)$ and delayed graft function $(p=0,012)$. Urological complications were not associated with donor, recipient and transplant factors. Lymphocele were associated with donor factors (non-traumatic brain death, $p=0,034$, asystoly and hypotension, $p=0,077, B M I, p=0,061$, presence of glomerular sclerosis at zerotime biopsy, $p=0,030)$, re-transplantations $(p=0,092)$ and ATG use $(p=0,094)$. Graft loss and patient survival during the follow-up period were not associated with mentioned surgical complications.
\end{abstract}

Conclusions. Post-transplant surgical complications are associated with donor condition but without impact on three-year graft and patient survival.

Key words: surgical complications, renal transplantation, donor factors, transplantation outcomes.

\section{INTRODUCTION}

Surgical complications (SC) after renal transplantation (RT) remain one the main concerns in transplantation (8). By the same time there are two clear tendencies in current transplantation: increasing age and co-morbidity in RT recipients leading to overload in "waiting list" and increasing shortage of donor organs, and expansion of the criteria for deceased donation in order to increase the number of donor organ available for transplantation (2). However, both tendencies may be associated also with increased risk of complications after RT, leading to worse transplantation outcomes, longer hospital stay after transplantation, and, accordingly, higher costs of treatment.

\section{AIM OF THE STUDY}

The aim of our study was to evaluate the association of surgical complications needing additional surgical interventions in the early posttransplant period with the condition of deceased donors, graft quality, recipient and transplantation factors, and to determine the impact of surgical complications on later posttransplant outcomes.

\section{MATERIALS AND METHODS}

Study included 202 consecutive deceased donor renal transplantations performed in a single center from 01.01.2004 till 31.12.2006. Patients were divided into four groups according to the presence of SC in the early posttransplant period (till the discharge from hospital): group A $(n=145)$ - without complications (control group); group B $(n=18)$ - with urological complications; group $C(n=27)$ - with vascular complications, such as bleeding and/or hematomas; group $\mathrm{D} \quad(\mathrm{n}=26)$ with lymphocele formation. In 12 cases there was a combination of two or three mentioned complications, and these patients were included into two or all three "complication" groups according to the complications observed.

We analyzed the rate of SC needing re-operations and surgical interventions in the early posttransplant period in association with donor factors (demographical and clinical factors, laboratory tests, histological findings at donor "zero" biopsy), as also recipient and transplantation factors (recipient age, gender, dialysis modality, cold ischemia time, vascular reconstructions at back-table surgery, etc.). Patients were followedup for three years in order to evaluate the impact of surgical complications on later posttransplant outcomes. All operations were performed by authors using the same organ explantation and transplantation techniques with retroperitoneal graft positioning in recipient (intraperitoneal approach and graft positioning in 2 cases of third transplantation). Donor kidney "zero" biopsies were performed at donor operations by "Truecut" method. Organ conservation was performed by histidine-tryptophan-ketoglutarate solution (HTK). 
Data was collected from transplantation center surgical, transplant coordination and ambulatory follow-up data-bases and records.

Statistical analysis was performed by SPSS 13,0 program (SPSS Inc.). Parametric features are shown as mean \pm standard deviation. Groups were compared using $\chi^{2}-$ tests for non-parametric and ANOVA test for parametric variables. Results were considered statistically significant for $p<0,05$.

\section{RESULTS}

SC that needed re-operations were observed in 57 patients $(28,2 \%)$, and in 12 of them $(5,9 \%)$ there was a combination of two or all three complications. Vascular complications (mainly bleeding and hematoma formation), were observed in 27 cases $(13,4 \%)$, urological (urine leak and ureteral stenosis) - in 18 cases $(8,9 \%)$, symptomatic lymphocele formation needing repeated punctions and drainage operations in 26 cases $(12,9 \%)$. Results of comparison of each "complication" group with the control group are shown in table 1 .

Vascular complications showed statistically significant association with the presence of glomerular sclerosis in kidney graft (observed in 18,5\% compared with $5,5 \%$ in control group, $p=0,034)$, as also tendency to be associated with higher donor BMI $(27,0 \pm 5,0$ vs. $25,4 \pm 3,8$ in control group, $p=0,067)$. This complication was also more frequently observed in elderly recipients, however, analysis failed to show statistical significance $(p=0,090)$. In postoperative period vascular complications were associated with the development of delayed graft function $(29,6 \%$ vs. $10,3 \%$ in control group, $p=0,012$ ), as also with urological complications (combination of those two complications was observed in 7 cases, $p=0,004$ ).

Urological complications failed to show statistical association with analyzed factors. The tendency of more frequent development of this complications was observed in transplantations from donors with high BMI $(27,1 \pm 5,3$ vs. $25,4 \pm 3,8$ in control group, $p=0,098)$. In posttransplant period urological complications were more frequently observed in patients who had delayed graft function $(p=0,050)$.

Lymphocele formations were associated with nontraumatic causes of brain death in donors $(p=0,034)$ and presence of glomerular sclerosis in "zero" biopsies $(p=0,030)$. Lymphocele formations were associated, however, without statistical significance, with higher donor BMI $(p=0,061)$ and hemodynamic disturbances in donors prior or during organ explantation operations $(p=0,077)$, as also with re-transplantations $(p=0,092)$ and with the use of antithymocyte globulin (ATG) for induction immunosuppression $(p=0,094)$. In posttransplant period lymphocele formations were relatively more frequently observed in patients with delayed graft function $(p=0,074)$.

\section{DISCUSSION}

Donor organ shortage is one of the main problems in transplantation that leads to more aggressive use of expanded criteria for deceased donation (2). The same situation is observed in Latvia (10), and it became of especial importance during the previous years, when economical crisis resulted in massive emigration of able-bodied population, increase in the number of objections for mostmortem donation associated with public negativism and mistrust, increase in the number of chronically ill persons due to inability to receive valuable medical help in time.

The use of ECD is associated with poorer posttransplant outcomes such as immediate graft function, duration of hospital stay and increased costs of treatment after transplantation (6).

SC in RT are rather frequent (near $30 \%$ of all cases) and could be associated with many factors: the use of anti-coagulation therapy in pre- and posttransplant period, immunosuppression and associated problems with increased risk of infections and retarded healing, disturbances caused by uremia and chronic dialysis, etc. $(1,3,5)$.

In this study we showed that some donor factors are associated with higher risk of the development of surgical complications. One of the main factors is the presence of glomerular sclerosis in donor kidneys, associated with increased risk of bleeding and development of hematomas and lymphocele in posttransplant period. In our earlier study we have shown that the presence of interstitial and glomerular sclerosis in donor kidneys is associated with worse early posttransplant outcomes (7). Unfortunately, it is still impossible to organize immediate histological analysis in order to get information on the quality of recovered kidneys prior to transplantation, therefore "zero" biopsies have only retrospective value. This one of our main problems, regarding the fact that histological finding are of highest value for appropriate organ allocation $(4,9)$.

Another factor associated with relatively higher incidence of surgical complications is high donor BMI that could be explained both by difficulties during the organ recovery operation and higher incidence of nephrosclerosis in overweight donors (7).

Relatively higher incidence of vascular complications in elderly recipients is more likely related with initially poorer vascular condition in these recipients (atherosclerosis, arterial stiffening). Higher frequency of lymphocele in transplantations from donors with hemodynamic disturbances may be explained by the necessity to perform rapid organ recovery operation and associated risk of traumatism of lymphatic vessels in grafts. Lymphocele complications after ATG treatment may have several reasons, such as hypocoagulation, retarded healing, etc.

All complications were associated only with the development of delayed graft function and failed to show association with increased risk of graft losses and patient deaths during the next three years after transplantation. Regarding this fact, we can conclude 
that transplantation from ECD is a valuable source of donor organs, especially for transplantation in patients with limited life expectancy; however, further follow-up is needed to determine the impact of such complications on later posttransplant period.

\section{Conclusions}

SC in early postoperative period are associated with the use of ECD, however, without major impact on first 3 years after transplantation. ECD are a valuable source of donor organs, especially for transplantation in patients with limited life expectancy.

\section{Conflict of interest: None}

\section{REFERENCES}

1. Akbar SA, Jafri SZH, Amendola MA, Madrazo BL, Salem R, Bis KG. Complications of Renal Transplantation // RadioGraphics, 2005; 25: 13351356

2. Delmonico FL, Sheeny E, Marks WH. Organ donation and utilization in the United States, 2004 // Am J Transplant, 2005; 2: 862-873

3. El Atat R, Derouiche A, Guellouz S, Gargah T, Likhoua R, Chebil M. Surgical complications in pediatric and adolescent renal transplantation // Saudi J Kidney Dis Transpl, 2010; 21: 251-257

4. Escofet $\mathrm{X}$, Osman $\mathrm{H}$, Griffiths DF, Woydag S, Adam Jurewicz W. The presence of glomerular sclerosis at time zero has a significant impact on function after cadaveric renal transplantation // Transplantation, 2003; 75: 344-346
5. Hernandes D, Rufino M, Armas S, Gonzales A, Gutierrez P, Barbero P, Vivancos S, Rodriguez C, Rodriguez de Vera J, Torres A. Retrospective analysis of surgical complications following cadaveric kidney transplantation in the modern transplant era // Nephrol Dial Transplant, 2006; 21: 2908-2915

6. Jushinskis J, Trushkov S, Bicans J, Suhorukov V, Shevelev V, Ziedina I, Rozental R. Risk factors for the development of delayed graft function in deceased donor renal transplants // Transplant Proceed, 2009; 41:746-748

7. Jushinskis J, Malcev A. Donor kidney biopsy results as a predictive factor for early graft function // Organs Tissues \& Cells, 2009; 12: 55-56

8. Karam G, Maillet F, Braud G, Battisti S, Hétet JF, Glémain P, Le Normand L, Bouchot O, Rigaud J. Surgical complications in kidney transplantation // Annales d'urologie, 2008; 41: 261-275

9. Mazzuccol G, Magnani C, Fortunato M, Todesco A, Monga $\mathrm{G}$. The reliability of pre-transplant donor renal biopsies (PTDB) in predicting the kidney state. A comparative single-centre study on 154 untransplanted kidneys // Nephrol Dial Transplant, 2010; 25: 3401-3408

10. Trushkov S, Jushinskis J, Suhorukovs V. Enhanced criteria donors: Impact of cold ischemia time in "old-to-old" kidney transplantation // Acta Chirurgica Latviensis, 2005; 5: 14-16 
Table 1. Comparison of groups

(* $-p$ value compared with control group; NC - no complications; UC - urological complications; VC - vascular complications; LC - lymphocele complication; BMI - body mass index; NTD - non-traumatic cause of brain death; ICU - intensive care unit; S-Crea - serum creatinine; S-Urea - serum urea; Hgb - hemoglobin; Hct - hematocrit; Leu - leucocytes; IS - interstitial sclerosis; GS - glomerular sclerosis; PD - peritoneal dialysis; HD - hemodialysis; Re-TX - re-transplantations; CIT - cold ischemia time; ATG - antithymocyte globulin; DGF - delayed graft function; AR - acute rejections)

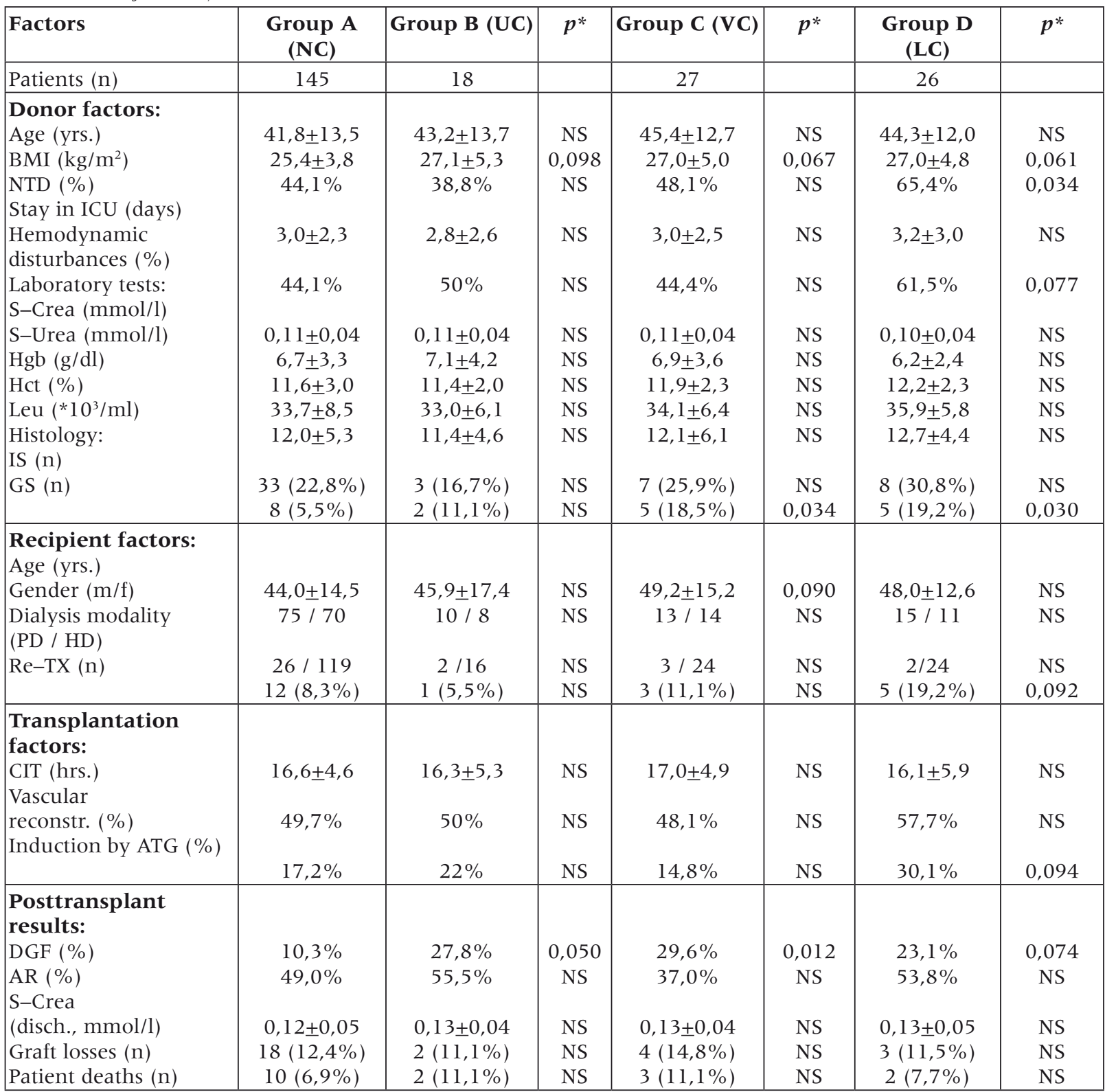

\section{Address:}

Janis Jushinskis

Paul Stradins University Hospital,

Latvian Transplantation Center

Pilsonu str. 13, Riga, LV-1002, Latvia.

e-mail: jushinskis@transplantation.lv 